\title{
HABERMAS CONTRA EL HIPERRACIONALISMO: APUNTES DEL DEBATE HABERMAS-LUHMANN A PROPÓSITO DEL TÓPICO MARXISTA DE LA IDEOLOGÍA
}

\author{
ENRIQUE SOTOMAYOR \\ Pontificia Universidad Católica del Perú
}

Sumilla: El presente trabajo plantea una revisión de la forma en que Jürgen Habermas retoma la tradición crítica del marxismo respecto del tópico de la ideología. Luego, y a partir de la caracterización del pensamiento de Habermas respecto del avance de la racionalidad instrumental (en detrimento de la razón comunicativa), afrontaremos el debate que han sostenido Habermas y Niklas Luhmann en el seno de la teoría social alemana. Finalmente, la tercera sección se centra en mostrar las diferencias en la concepción sobre el lugar del derecho en la sociedad, a partir de los marcos teóricos de Habermas y Luhmann.

Palabras clave: Teoría crítica, ideología, filosofía del derecho, acción comunicativa, teoría de sistemas.

Abstract: This paper presents a revision of the way in which Jürgen Habermas returns to the critical tradition of Marxism regarding the topic of ideology. After the characterization of Habermasian thinking regarding the progress of instrumental rationality (at the expense of communicative reason) it confronts the debate between Habermas and Niklas Luhmann within German Social Theory. Finally, the third section focuses on showing the differences regarding the conception of the place of law in society, based on the theoretical frameworks of Habermas and Luhmann.

Keywords: Critical Theory, ideology, philosophy of law, communicative action, systems theory. 
En este trabajo vamos a analizar la relectura de la crítica de la ideología que realiza Habermas. Para ello utilizaremos su propia obra, así como las interpretaciones que sobre la misma han hecho, sobre todo, Larraín ${ }^{240}$ y Ricoeur ${ }^{241}$. Finalmente, para analizar el recorrido histórico por el que transita Habermas resultará necesario hacer alguna referencia a la Escuela de Frankfurt como corriente teórica de la cual Habermas recibe los insumos de su obra.

Sobre este último punto, y como el filósofo más importante de la llamada segunda generación de la Escuela de Frankfurt, Habermas recibió una fuerte influencia de Theodor Adorno. Así, a pesar de su formación en Gotinga con algunos docentes de inclinación conservadora e incluso nacionalsocialista ${ }^{242}$, Adorno vio en el joven Habermas a un nuevo elemento importante en el Instituto de investigación social. Wiggershaus en su recuento de la historia de la Escuela ha señalado que:

“(...) Habermas era un filósofo social cuyo interés estaba dirigido exactamente a lo que se incluía de manera más bien esquemática en los trabajos ensayísticos de Adorno y en las ponencias y discursos de Horkheimer: una teoría de la época presente, de la patología de la época moderna"243.

Estos intereses en Habermas, lo llevaron a retomar los trabajos de Marx sobre la ideología como una forma de dominación. En ese sentido, y ya en específico sobre la discusión en torno a la ideología, Larraín ${ }^{244}$ plantea la posibilidad de diferenciar dos momentos en la concepción de dicho concepto en la obra de Habermas: Un primer periodo que va hasta el año $198 \mathrm{I}$ en que se publica la versión alemana de la Teoría de la acción comunicativa, y una segunda etapa a partir de la publicación de dicha obra. Mientras que en la primera Larraín señala que podemos distinguir dos elementos básicos: a) el tránsito de un tipo de ideología propio del siglo XIX hacia una nueva, tecnocrática, posibilitada por el impacto de la ciencia y la tecnología, y b) el reemplazo de la teoría de la

240 Larraín, Jorge, El concepto de ideología, Santiago de Chile: LOM Ediciones, $201 \mathrm{I}$.

24I Ricoeur, Paul, Ideología y utopía, Barcelona: Gedisa, 2008.

242 Cf. Wiggershaus, Rolf, La Escuela de Fráncfort, Buenos Aires: Fondo de Cultura Económica; Universidad Autónoma Metropolitana, 2011, p. 673.

243 lbid., p. 672.

244 En lo sucesivo expondremos la postura de Larraín en torno al concepto de ideología en Habermas: cf. Larraín, Jorge, op. cit., pp. 109-139. 
conciencia por una nueva teoría de competencias comunicativas que implica un segundo cambio, aún más importante, cual es el paso de una filosofía de la conciencia hacia una filosofía del lenguaje; en el segundo periodo asistimos a un decrecimiento en la importancia del concepto de ideología, en pro de una conceptualización de la conciencia fragmentada, clave para situar las críticas que Habermas dirige contra Luhmann y sobre las que volveremos más adelante.

La síntesis que Habermas realiza solo puede ser entendida a partir del estudio de sus influencias teóricas. Así, Larraín propone que del marxismo, Habermas va a tomar la idea de ideología entendida como un concepto crítico y relacionado directamente con la legitimación de formas de dominación mediante una serie de constructos institucionales. Pero por otro lado, de la teoría crítica Habermas va a tomar la crítica a la razón instrumental, teorizada en extenso en los trabajos de Adorno, Marcuse y Horkheimer. Dicha razón instrumental estaría indisolublemente ligada al despliegue de una ciencia y tecnología cada vez más especializadas y que comienzan a jugar un rol ideológico en el capitalismo avanzado. Pero finalmente, podemos identificar un tercer elemento que constituye el aporte original de Habermas a la confluencia entre marxismo y teoría crítica: el rol de la comunicación con respecto a dos temas en concreto. Primero, la comunicación estará asociada al concepto de razón, el mismo que deberá ser ampliado para abarcar a la razón comunicativa (y a la estéticopráctica en la obra de madurez de Habermas). En segundo lugar, el nuevo elemento lingüístico incorporado por Habermas tiene un papel importante en la superación de la filosofía de la conciencia ${ }^{245}$.

Habermas aprecia que con el advenimiento del capitalismo por sobre el feudalismo se inició un segundo movimiento un tanto más imperceptible: la extensión cada vez mayor de un sistema de acción racional con arreglo a fines. Este sistema de acción racional se ha impuesto solo al costo de reducir los espacios en los que antes se desplegaba un sistema de acción comunicativa o de interacción simbólica ${ }^{246}$. Es en este punto en el que Habermas considera que los

245 Para una importante aproximación a este punto: cf. Always, Joan, Critical Theory and Political Possibilities: Conceptions of Emancipatory Politics in the Works of Horkheimer, Adorno, Marcuse, and Habermas, Westport, CT: Greenwood Press, 1995, p. 116 ss.

246 Sobre este punto, es necesario remitirse al análisis que Habermas hace de la obra de Marcuse y Weber: cf. Habermas, Jürgen, Ciencia y técnica como "ideología", Madrid: Tecnos, 2009, p. 56-58. 
análisis de Weber eran incorrectos, no era que el problema de la modernidad podía ser identificado con una racionalización burocratizante en expansión sino que más bien el problema era que un solo tipo de racionalidad comenzó a penetrar en ámbitos en los que debía ser la racionalidad moral-práctica la que primara ${ }^{247}$, la misma que se forma en base a consensos conseguidos en una esfera pública entendida como mundo de la vida ${ }^{248}$.

Este proceso de difícil percepción hizo que la dominación ideológica pasara a fundarse en la economía de mercado y su lógica medio-fin, así como en el principio de intercambio igualitario. Podríamos agregar en este punto e indicar que Habermas complementa y supera la visión de la base o infraestructura en Marx. En los análisis efectuados por este último autor, no teníamos una explicación, más allá del trabajo humano como praxis productiva, de porqué la economía se encontraba en la base mientras que otros aspectos institucionales de la organización de la sociedad aparecían como la superestructura ideológica de la misma. Entonces, si atendemos a la historia -paralela a la del desarrollo del capital-que se forma en torno a la racionalidad medio-fin contra la racionalidad comunicativa, notaremos que la explicación de la primacía de la base en los análisis de Marx se podría encontrar -sin que siquiera Marx lo notara- en la primacía de la razón instrumental en detrimento de la razón comunicativa.

Sin embargo, Habermas nota que esta lógica de desenvolvimiento del capitalismo ha cambiado a partir del siglo XX. Dos fenómenos concretos como el aumento de la intervención del Estado en la economía y la creciente interdependencia de la ciencia y la tecnología podrían explicar este cambio ${ }^{249}$. En ese sentido Larraín considera que se habría producido un paso desde

247 Cf. ibid., p. 53 ss.

248 Sobre el concepto de mundo de la vida, Habermas señala que: "Desde la perspectiva interna del mundo de la vida la sociedad se presenta como una red de cooperaciones mediadas comunicativamente. Yno es que de esta perspectiva queden desterradas todas las contingencias, todas las consecuencias no pretendidas, todas las coordinaciones fallidas y todos los conflictos. Pero lo que liga entre sí a los individuos socializados y lo que asegura la integración de la sociedad es un tejido de acciones comunicativas que sólo pueden tener buen suceso a la luz de tradiciones culturales, y no mecanismos sistémicos que escapen al saber intuitivo de sus miembros" (Habermas, Jürgen, Teoría de la Acción comunicativa, Madrid: Trotta, 2 v., 20I0, p. 632).

249 Cf. Habermas, Jürgen, Ciencia y técnica como “ideología”, p. 81. 


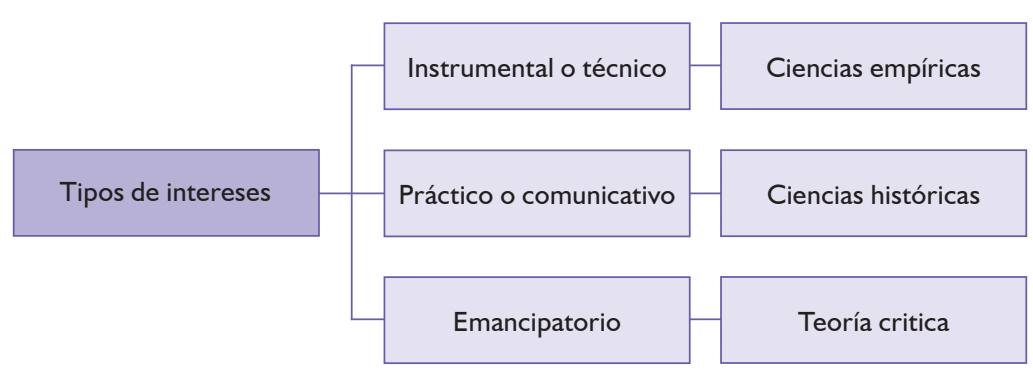

Figura I: Tipos de intereses y los tipos de ciencias que se corresponden a estos según Habermas (basado en la propuesta de lectura de Habermas realizada por Larraín) Fuente: Elaboración propia.

el capitalismo liberal clásico hacia una forma de capitalismo industrial en el que la legitimación ideológica del poder político se encuentra en el lenguaje técnico de la ciencia. Más aún, asistiríamos al surgimiento de una conciencia tecnocrática que despolitiza los problemas y justifica el ejercicio del poder por los expertos.

Latinoamérica no ha sido ajena a intentos de despolitización de este tipo. Un buen ejemplo ofrece Guillermo O'Donnel1 ${ }^{250}$, quien propuso una correlación entre la aparición de regímenes autoritarios y un lenguaje de técnicos en el continente, especialmente durante los años de dictaduras militares. En ese sentido, su teoría del autoritarismo burocrático se sostiene en la protección de un modelo de crecimiento de Industrialización por Sustitución de Importaciones por parte de una elite tecnócrata dispuesta a apoyar a las Fuerzas Armadas en golpes de estado frente a una creciente inestabilidad social (generada por el crecimiento sindical de las décadas anteriores). Este fenómeno tuvo lugar en países como Argentina o Chile luego de la caída del gobierno de Salvador Allende. Asimismo en el Perú tenemos antecedentes de esta forma ideológica de pensamiento en las reformas neoliberales de inicios de los noventa, en las cuales se debilitó el elemento democrático en pro de una respuesta técnicamente acertada desde la teoría microeconómica en boga (el marginalismo). 
La tecnificación creciente de nuevos ámbitos de las interacciones humanas esconde, como ya hemos visto, la diferencia entre dos tipos distintos de racionalidad: una que podemos llamar acción racional con arreglo a fines y una segunda, el aporte de Habermas, llamada acción comunicativa.

Pero a diferencia de una ideología tosca del tipo que Marx criticaba en la concepción del Estado en Hegel -pura necesidad especulativa, y más aún, falta de motivación en el caso del monarca hereditario ${ }^{251}$ - la ideología de tipo tecnocrático es más sutil, y por ello, difícil de criticar. Esto último se debe a que, estrictamente, no es mera ideología lo que se critica, sino que existen asideros cuantitativos y cualitativos, basados a su vez en resultados de pruebas inductivas en, por ejemplo, las ciencias sociales (en algunas ocasiones las escuelas empiristas de la sociología norteamericana se acercan mucho a este modelo ${ }^{252}$ ), que sustentan afirmaciones que aun así pueden ser calificadas como ideológicas en el nuevo sentido habermasiano. Por ello, para construir una crítica consistente a sus postulados es necesario que el teórico reconstruya distintos tipos de razón y racionalidad presentes en distintos estadios histórico-productivos, y a partir de ello construya una suerte de topología. De no hacerlo toda la teoría corre el riesgo de perderse en un racionalismo instrumental acusable más que todo por una suerte de provincianismo histórico (consistente en creer que el tipo de racionalidad que prima aquí y hoy fue la que primó y primará en todas las sociedades humanas).

Ello llevará a Habermas a asumir, según Larraín, un punto medio entre Marx y Marcuse. Contra Marx, Habermas sostendrá que las fuerzas productivas no son ni pueden ser siempre potencial de liberación, ello en la medida que su continuo crecimiento se ha hecho posible solo a costa de una creciente

25I Cf. Hegel, G.W.F., Principios de la filosofía del derecho, Barcelona: Edhasa, 2005, p. 370 ss. Como señala Ricoeur, esta forma de proceder abandonando las categorías de lo concreto: "Para Marx será el modelo del pensamiento ideológico, un movimiento que va desde la idea a la realidad y de desde la realidad hacia la idea" (Ricoeur, Paul, op. cit., p.69). Así, antes de identificar a un monarca con la forma del absoluto del despliegue de la institución estatal, Marx proclama la necesaria vuelta a las relaciones humanas que han hecho necesaria o útil la centralización de algún grado de poder. Podemos ver entonces que en Marx desaparece el fin inmanente del Estado y de sus poderes -en especial el del príncipe o monarca hereditario- y aparece una necesidad exterior de organizar una sociedad civil.

252 Aquí podríamos ubicar, por ejemplo, a algunas teorías empiristas sobre los movimientos sociales, tales como la de la acción colectiva o la de los nuevos movimientos sociales: cf. Gamson, William A., The strategy of social protest, Homewood: The Dorsey Pres, 1975. 
dependencia del progreso científico y técnico. Si la función ideológica de la conciencia tecnocrática era precisamente la de legitimar el poder político ya vemos en qué sentido las fuerzas productivas no son siempre potencial emancipatorio tal como Marx parecía prever a partir de la lectura del Prefacio a la contribución de la economía política ${ }^{253}$ y de algunos pasajes de El Capital.

Asimismo y contra Marcuse, Habermas señalará que el pesimismo de éste respecto de la racionalidad tecnológica le impide ver que esta también es una fuerza productiva progresista y de un enorme potencial emancipatorio ${ }^{254}$. Es decir que, contra Marcuse, Habermas más bien recupera el optimismo de Marx respecto de las fuerzas productivas. Pero, ¿en qué medida la racionalidad tecnológica puede ser progresista? Pues en la medida que esta suele estar relacionada, en su despliegue, con el progreso de la ciencia y la tecnología y, con ello, de las fuerzas productivas. Con dicho despliegue constante se amenaza el marco institucional vigente, el mismo que aparece como ideológico en el sentido más tradicional de Marx, es decir como una cadena que se ubica en las relaciones de producción y que limita el despliegue -ya fácticamente posible según las circunstancias históricas- de las fuerzas productivas. De esta manera, la ciencia solo se hace ideología cuando está conectada con intereses instrumentales que tienden a reducir la esfera de los intereses comunicativos (se produce una suerte de desbalance entre ambos).

Ahora bien, icómo logramos el objetivo de desterrar el ámbito ideológico presente en la ciencia del capitalismo tardío? En el verano del 1965 Habermas impartió clases como sucesor de Horkheimer y dedicó sus clases al tema del Conocimiento e interés ${ }^{255}$. La teoría crítica que en esta obra esboza Habermas contiene los elementos esenciales de lo que podemos entender por su concepto

253 En uno de los pasajes más célebres de dicha obra, Marx señala que "No es la conciencia del hombre la que determina su ser sino, por el contrario, el ser social es lo que determina su conciencia. Al llegar a una fase determinada de desarrollo las fuerzas productivas materiales de la sociedad entran en contradicción con las relaciones de producción existentes o, lo que no es más que la expresión jurídica de esto, con las relaciones de propiedad dentro de las cuales se han desenvuelto hasta allí. De formas de desarrollo de las fuerzas productivas, estas relaciones se convierten en trabas suyas, y se abre así una época de revolución social" (Marx, Karl, "Prólogo a la contribución a la crítica de la economía política", disponible en: http://www.marxists.org/ espanol/m-e/l850s/criteconpol.htm).

II4 255 Cf. Habermas, Jürgen, Conocimiento e interés, Buenos Aires: Taurus, 1982. 
de ideología, tal como también lo entendió Ricœur. A partir de dicha obra, Habermas argumentará que se logra desterrar el ámbito ideológico mediante la repolitización de los procesos deliberativos por los cuales se toman decisiones, es decir, con una teoría de democracia deliberativa procedimental ${ }^{256}$, a la vez que mediante un levantamiento de las restricciones a la racionalidad dialógica entendidas como una apertura del debate en el que antes solo tenían voto autorizado los técnicos.

La figura I muestra los tipos de intereses ${ }^{257}$ que se corresponden con el desarrollo de distintas áreas de conocimiento según Habermas. En ese sentido, dicho autor va a llamar la atención sobre el descuido en que incurrió no solo el marxismo sino también parte de la teoría critica, entre ellos, Marcuse y Horkheimer en no distinguir entre intereses instrumentales y comunicativos. Así, en la medida que Marx no pudo identificar dicha diferencia, no pudo notar que los conflictos respecto de la ideología no se producían en toda la estructura de la base sino solo en aquella parte que tiene que ver con las relaciones de producción.

Como señala Ricoeur sobre este punto:

"Como veremos, Habermas sostiene que ciertas ciencias corresponden a ciertos intereses. El interés de controlar y manipular corresponde a las ciencias empíricas; el interés por la comunicación corresponde a las ciencias históricas e interpretativas; $y$ el interés en la emancipación corresponde a las ciencias sociales críticas tales como el psicoanálisis"258.

Para Ricoeur, Habermas complementa le dicotomía base/superestructura señalando que ahí donde en la base existe distinción entre fuerzas productivas

256 Para una crítica a una suerte de dialogismo excesivo en Ética del discurso: notas sobre un programa de justificación filosófica de Habermas, cf. Nino, Santiago, La constitución de la democracia deliberativa, Barcelona: Gedisa, 2003, p. 157 ss. Aquí Nino plantea que la mera deliberación en democracia no garantiza que los acuerdos a los que se arriben tengan superioridad moral a aquellos impuestos unilateralmente por un dictador. En esa medida, resulta necesario efectuar un complemento a la teoría deliberativa de Habermas, y la misma consiste en una serie de preceptos procedimentales que reajustan la democracia deliberativa y funcionan como filtros a los argumentos que se pueden o no usar en el debate público.

257 El sentido en el que aquí Habermas usa los términos interés o intereses, es distinto al sentido en el que utiliza la noción de interés en su análisis de la dicotomía entre intereses materiales e intereses ideales en Max Weber. Para esta última acepción del término, cf. Habermas, Jürgen, Teoría de la Acción comunicativa, pp. 229-237.

258 Ricoeur, Paul, op. cit., p. 243. 
y relaciones de producción, el marco institucional, legal y de interacciones propio de las relaciones de producción, necesita a su vez de una estructura de interacción simbólica y dialógica. Este es el punto que el marxismo habría obviado hasta entonces, el ver en la estructura de la base simplemente a la razón instrumental y no una mezcla de razón dialógica y acción racional con arreglo a fines ${ }^{259}$. ¿Qué llevó a ese extravío del marxismo? Larraín sostiene que el mismo es compartido por todas las filosofías tradicionales de la conciencia, mediante las cuales el sujeto se relaciona monológicamente con el medio en el cual interactúa. En ese sentido el error de Marx no sería solo suyo, sino que se habría replicado en la racionalidad instrumental de Adorno y Horkheimer, y, en general, en toda la historia de la filosofía occidental. Este último punto lleva a Ricoeur a concluir que la ideología solo se da en el plano de las relaciones de producción y no en el de las fuerzas productivas ${ }^{260}$. Este punto queda claro con el siguiente pasaje:

"La objeción de Habermas a la que este vuelve continuamente, es la de que Marx redujo el concepto de actividad a la producción. Así quedó quebrantada la extensión del concepto. Si bien Marx resolvió el problema de la síntesis por el trabajo, redujo el alcance de su descubrimiento al identificar el trabajo con la mera acción instrumental”261.

Pero existe otro elemento con el cual Habermas comienza a formar un nuevo concepto de ideología más amplio que aquel que contempla al ámbito de la praxis productiva frente a la estructura del pensamiento de una época. Podemos sostener que Habermas se vio beneficiado, para construir su postura crítica al positivismo como ideología, de las disputas que en las décadas del cincuenta y sesenta sostuvieron Karl Popper y Theodor Adorno. En dicho debate, Popper concebía que el gran proyecto de extrapolar el método inductivo ${ }^{262}$ propio de las ciencias naturales hacia las ciencias sociales constituía

259 Cf. ibid., p. 247.

260 "La ideología se da solo en el nivel de las relaciones de producción, no en el de las fuerzas de producción [fuerzas productivas]" (ibid.).

261 lbid., p. 246.

262 Para resaltar el nivel de penetración e importancia del debate en torno a la extensión aplicativa de métodos deductivos e inductivos para el tratamiento de problemas de disciplinas de ciencias sociales y humanidades, podemos mencionar que el filósofo del derecho Luigi Ferrajoli ha utilizado la distinción para defender una metaética anticognitivista en el derecho. Una de las consecuencias de esta asunción, es que el discurso de disciplinas tales como el derecho no puede tener una pretensión de verdad sino tan solo de racionalidad (cf. Ferrajoli, Luigi y Juan 
una forma de cientificismo que partía de un mal entendido. A pesar de la adhesión de Habermas a la postura de Adorno en este debate, el mismo Habermas pareció encontrar en las críticas de Popper, los elementos que ayudaron a la formulación final de su teoría de la acción comunicativa que aparecería décadas después ${ }^{263}$. Este era, en alguna medida, el punto en el que los análisis de Marx, Adorno y Horkheimer fallaban, los mismos partían de una filosofía tradicional del sujeto que olvidaba que estos se relacionan con el mundo objetivo de manera colectiva. Ello, contra la pretensión cientificista, significa que existe por lo menos un ámbito de las interacciones sociales en el que las formas de coordinación y entendimiento tienen un papel central, y que ese lugar es el de la acción comunicativa ${ }^{264}$. Ello es así, además, debido a que la tecnificación de crecientes campos de la interacción humana tiene consecuencias en la fragmentación de las formas de comunicación. De este modo, podemos entender que la ideología de la sociedad del siglo XX corresponde a situaciones en las que una comunicación sistemáticamente distorsionada por la predominancia de un lenguaje técnico fragmentario (Larraín agrega que la distorsión también se produce por violencia, censura o represión) -propio de la razón instrumental de pretensiones cientificistas-impide la emergencia de consensos prácticos entre los integrantes de una sociedad.

De este punto Larraín extrae dos consecuencias: Por un lado, concluye en que la ideología tecnocrática no solo se vincula a intereses particulares sino que también afecta a los intereses emancipatorios de la humanidad en su conjunto. ¿De qué manera? Mediante la fetichización de la ciencia de una forma tal que destierra campos enteros de la deliberación y el consenso, a la vez que los oculta de estas formas de entendimiento. Pero por otro lado, esta forma de proceder llevaría a Habermas a tratar la problemática de la ideología de forma similar a como el psicoanálisis trata el problema de la neurosis. Contra la clásica postura de Weber recelosa de la racionalización y burocratización

Ruiz Manero, Dos modelos de constitucionalismo: una conversación, Madrid: Trotta, 2012, p. 58 ss).

263 Cf. Wiggershaus, Rolf, op. cit., p. 709 ss.

264 Sobre este punto de la disputa el mismo Habermas concibió un dialogo sin coacción como condición de posibilidad en las ciencias sociales. Este modo de proceder estaría alejado del método inductivo y del modo de proceder de las ciencias naturales (cf. Habermas, Jürgen, “Dogmatismo, razón y decisión. Teoría y praxis en la civilización científica”, en: Teoría y Praxis, Madrid: Tecnos, 2000, p. 288). 
social, Habermas sostendrá precisamente lo contrario, a saber, que el problema compartido entre la ideología a nivel a agregativo y la neurosis a nivel individual es el de la racionalización.

Sin embargo para Ricoeur el uso extensivo del psicoanálisis como técnica de análisis social por parte de Habermas no es tan evidente. Es más, a pesar de las numerosas páginas que Habermas dedica al tema en Conocimiento e interés, nunca se hace alguna alusión expresa a la posibilidad de extrapolar el psicoanálisis a la crítica de la ideología. Aún con estas reservas, Ricoeur 265 identifica cuatro puntos en los que la transferencia de metodología de los modelos resulta plausible. Un primer punto tiene que ver con que tanto el tratamiento de procesos de neurosis, así como ideológicos, implica procesos de autorreflexión y autocomprensión por los cuales el sujeto o analista detienen el desenvolvimiento de un proceso para ver si es que algo no está fallando en el mismo. Un segundo punto de convergencia es que las deformaciones tanto en el psicoanálisis como en la ideología se dan a un nivel de emancipación. Así, en la medida que los problemas de racionalización implican procesos de interrupción de comunicación, "nos vemos obligados a hablar hasta de la lucha de clases desde el punto de vista de la comunicación"266. Esta lucha de clases, sin embargo, ya no está relacionada a la figura de sectores antagónicos enfrentados por la distribución de los medios de producción, sino con una interrupción del proceso de comunicación que lleva a extrañamientos entre las personas. La tecnificación en ese sentido llevaría a la ruptura de lenguajes compartidos, y con ello, al debilitamiento del mundo de la vida como espacio compartido y sustrato del debate público. La tercera aplicación que encuentra Ricoeur consiste en que tanto el psicoanálisis como la ideología tratan contra deformaciones sistémicas, $y$ en esa medida es necesario que se restablezca la comunicación, lo cual implica que el problema no es, ni siquiera, la falta de esta. El paralelismo final que Ricoeur traza entre psicoanálisis y critica de la ideología tiene que ver con la extracción de modelos teóricos con variedad de aplicaciones de análisis, uno de los cuales implica la reificación de la sociedad que es tratada como un paciente neurótico. Entonces el teórico critico o psicoanalista que desentraña el mecanismo por el que opera la ideología debe comparar situaciones de comunicación distorsionadas a la luz de una

265 Cf. Ricoeur, Paul, op. cit., pp. 266-267. 
situación ideal del habla. ¿De dónde extraer ese modelo normativo? Habermas planteará que ese modelo corresponde con una situación ideal del habla en la que la deliberación no está fragmentada ni aquejada de violencia o censura. Solo contra este modelo podemos juzgar las pretensiones de corrección de una situación dada.

Cannon señala que:

“(...) Habermas's subsumes 'practical reason' beneath its 'functional' other. In order to remedy this Habermas returns to his Kantian-inspired 'exclusion model' in which the technically practical realm of labour is dirempted from the morally practical realm of communication. The resulting uncoupling of system and lifeworld reaffirms the opposition between an instrumental form of labour and an ethically charged form of communication" 267 .

Sin embargo, sobre la capacidad que ve Habermas de que las situaciones de distorsión en la comunicación sean corregidas sin apelar a una salida revolucionaria o emancipadora, Larraín ${ }^{268}$ ve un optimismo habermasiano en el capitalismo tardío para salvar la latencia del conflicto de clases que se ha hecho conflicto de clases a propósito de la comunicación. Es por ello que se podría calificar a Habermas como un pensador de la racionalidad tecnológica; es decir, de un tipo tal, que considera que el sistema mismo está en aptitud de eliminar las posibilidades de crisis a su interior mediante tres mecanismos específicos: Encontrando soluciones técnicas a los problemas de distorsión en la comunicación, integrando a las clases sociales rebeldes y evitando explosiones políticas.

Este último punto nos lleva, al igual que a Larraín, al análisis final de algunas de las continuidades y rupturas en la obra de madurez de Habermas, y en concreto al periodo que sigue a la publicación de la Teoría de la acción comunicativa de 198I. Aquí Habermas abandonará el uso extensivo del concepto de ideología tal como otras corrientes lo habían comenzado a hacer (el posmodernismo

267 Cannon, Bob, Rethinking the Normative Content of Critical Theory: Marx, Habermas, and beyond, New York: Palgrave, 200I, p. 124.

268 Cf. Guy, Pierre, La teoría del ciclo largo de Mandel y la historia económica, disponible en: http:// www.izt.uam.mx/economiatyp/numeros/numeros/primera_epoca/04/articulos_pdf/4_7_la_teoria_ciclo.pdf 
o el mismo postestructuralismo de Foucault, por ejemplo). En este periodo Habermas parece considerar a la ideología como falsa conciencia que opera ocultando, como un modelo solo vigente respecto de los sistemas totalizantes del siglo XIX. En contraposición a este concepto de ideología, podríamos identificar uno nuevo que ya estaba esbozado en la obra anterior de Habermas, el de una conciencia fragmentada que opera impidiendo el alcance de síntesis integradoras dotadas de legitimidad social. El problema entonces no es que exista una acción racional con arreglo a fines que opera en base a objetivos externos al sujeto, sino que dicha razón se encuentra colonizando el mundo de la vida (es decir que asistimos a un avance de la racionalidad cognitivoinstrumental). Es por ello que en el capitalismo industrial de occidente estaría operando un patrón selectivo de racionalización pues la racionalidad moralpráctica y estético-práctica va dejando su lugar en contra del avance de la racionalidad de tipo instrumental.

Podríamos decir que la obra posterior de Habermas en la que desarrolla las consecuencias de su teoría de acción comunicativa se dedican precisamente a construir un estándar normativo a partir del cual se puede juzgar la pretensión de validez de una situación de comunicación fáctica. Así, en el derecho, por ejemplo, esta pretensión se ha visto reflejada en su obra Facticidad y validez ${ }^{269}$.

\section{§ 1. La teoría de sistemas de Niklas Luhmann: una caracterización}

Para poder delimitar las razones por las cuales Habermas va a considerar que la teoría de sistemas de Niklas Luhmann representa una forma de ideología hiperracionalista, resulta indispensable comenzar esta sección a través de una caracterización de dicha teoría en el modo que la concibe el teórico social alemán Niklas Luhmann. Esta exigencia se impone en la medida de que existe una multiplicidad de teorías de sistemas que ponen énfasis en distintos elementos tanto estructurales como prácticos; y que incluso se valen de distintos marcos teóricos para sus construcciones. Sin embargo, el acercamiento no podrá ser exhaustivo debido a que la finalidad es solamente la de en términos de teoría del discurso, Madrid: Trotta, $200 \mathrm{I}$. 
mostrar de qué manera Habermas va a argumentar que la teoría de sistemas de Luhmann es ideológica; y con ello la bibliografía a analizar, en el caso de Luhmann, será limitada.

Pues bien, en primer lugar podríamos señalar que la teoría de Luhmann puede ser clasificada como una de tipo sistémica funcional-estructural, mientras que la de uno de sus más importantes referentes, Talcott Parsons es de tipo estructural-funciona ${ }^{270}$. La diferencia entre ambas variantes puede ser encontrada en el énfasis. Así, mientras que la teoría de Parsons busca desentrañar los mecanismos operativos de la estructura que se comporta de determinada manera, a Luhmann solo le va a llamar la atención el aspecto del funcionamiento de dichas estructuras ${ }^{271}$. Aquí podemos notar una fuerte influencia de la cibernética en el pensamiento de Luhmann. En efecto, en algunos de sus análisis como Law as a social System ${ }^{272}$ Luhmann parece optar por un modelo de black box o caja negra para analizar el funcionamiento del sistema legal.

En dicho modelo, según Bullesbach "No se precisan (...) imprescindiblemente conocimientos acerca de la estructura interna del sistema"273. Es decir, más que las lógicas por las que los sistemas operan en su interior, que Luhmann considerará altamente tecnificadas y sofisticadas, interesa el producto visible en el output de lo que el sistema procesó desde un inicio (input). Por otro lado, existe en Luhmann un abandono progresivo de las categorías de la cibernética en la medida que asistimos al desarrollo de su obra. García Amado se ha referido a dicha evolución en estos términos:

270 Cf. Hassemer, Winfried (ed.), El pensamiento jurídico contemporáneo, Barcelona: Debate, 1982, p. 322.

27I Sobre el poco énfasis e importancia que Luhamm pone sobre el funcionamiento interno de un sistema, él mismo señala: "The description of a system as autopoietic, as autonomous, as operationally closed, refers to the network of its operations and not to the totally of all empirical conditions, that is, the world. The question is not how a system can maintain itself without any environmental support. Rather, it is what kind of operations enable a system to form a self-reproducing network which relies exclusively on self-generated information and is capable of distinguishing internal needs from what it sees as environmental problems" (Luhmann, Niklas, "Operational clousure and structural coupling: the differenciation of the legal system", en: Cardozo Law Review, XIII, p. 1420).

272 Cf. Luhmann, Niklas, “Law as a social system”, en: USA: Northwestern University Law Review, LXXXIII, No. I-2, 1989, p. I36-I50.

273 Bullesbach, Alfred, "Enfoques de teoría de sistemas", en: Hassemer, Winfried (ed.), op. cit., p. 320. 
"Una de las dificultades de la muy voluminosa serie de los escritos de Luhmann suscita es la de averiguar si en ellos se contiene una línea evolutiva estable o si, por el contrario, hay rupturas o giros importantes. La cuestión se plantea especialmente a partir de comienzos de los ochenta, de las nociones de autopoiesis y sistemas autorreferenciales, conceptos provenientes de la biología y debidos a Varelay Maturana"274.

Este abandono también alcanzaría a la gran influencia de su obra temprana: Talcott Parsons, razón por la cual Habermas dirige su crítica en su Teoría de la acción comunicativa no contra Luhmann en tanto representante de la teoría de sistemas sino contra el mismo Parsons. La razón de este cambio de objetivo de la crítica habermasiana responde, en alguna medida, a un giro conceptual del mismo Luhmann, que desde la perspectiva de Habermas habría incurrido, de manera mucho más clara que Parsons, en el mismo error que Popper criticaba al cientificismo, el de extender el método inductivo de las ciencias naturales hacia las ciencias sociales ${ }^{275}$. Pero por otro lado, la teoría de sistemas luhmanniana también se diferencia de la llamada teoría de sistemas comple$j^{2} \mathrm{~s}^{276}$ en la medida que mientras una busca articular distintos conocimientos de cada subsistema tecnificado del conocimiento moderno en una serie de categorías unitarias que faciliten la elaboración de un lenguaje común, la teoría de Luhmann más bien va a proclamar que la especialización funcional es el producto natural de la evolución de las sociedades modernas. En la medida que dichas sociedades asisten a una complejización -y con ello, a una caotización del mundo en muchas variables dispersas- se hacen necesarios una serie de conocimientos que se articulan internamente en base a oposiciones binarias que ayudan a reducir la complejidad del mundo.

Mientras que el objetivo de la Teoría de sistemas complejos puede ser entendida en los siguientes términos:

274 García Amado, Juan Antonio, La filosofía del derecho de Habermas y Luhmann, Bogotá: Universidad Externado de Colombia, 1997, p. 185.

275 Cf. Luhmann, Niklas, "En el ocaso de la sociología crítica", en: Revista Sociológica. Perspectivas y problemas teóricos de hoy, No. 20 (1992), Universidad Autónoma Metropolitana, Departamento de Sociología, México, pp. 186-194. También cf. Arnold, Marcelo, Habermas y Luhmann: continuidades y discontinuidades, disponible en: http://www.marceloarnold.cl/images/ habermas.pdf

276 Cf. Earls, John, Introducción a la teoría de sistemas complejos, Lima: Fondo Editorial PUCP, 2011. 
"Lo que hace la ciencia de la complejidad [contra el paradigma científico clásico] es revertir este proceso y juntar todas las disciplinas en la búsqueda de principios universales de funcionamiento de los sistemas sin importar que sean sistemas sociales, biológicos, químicos, etcétera"277.

El objetivo de la teoría de sistemas será completamente distinto, pues en lugar de buscar la complejizacion de los problemas, se busca su diferenciación y tratamiento en parcelas distintas de conocimiento. La diferenciación funcional creciente, más que un síntoma indeseable de la modernidad tecnificada, es la expresión de la voluntad del hombre de simplificar el caos de las enormes extensiones de conocimiento que necesitan coordinación. En un ejemplo simple sobre un sistema mecánico que trabaja sobre el código binario calor/ frio, Herget señala:

"A system is an operating entity that functions through its own structures and processes. In our example the system consists of the totality of components that systematically perform the function of heating (the thermostat, the furnace, the electrical wiring, etc.) plus their dynamic operations. Everything else is the environment of the system. This distinction between system and environment is an important basic concept in Luhmann's thinking" 278 .

Aquí vemos un punto de alejamiento de Luhmann respecto de la cibernética o del modelo de black box al que antes hemos hecho referencia. Mientras que en la teoría de sistemas complejos se pueden construir patrones explicativos pues se trabaja en base a una serie de categorías globales (una de las cuales es la del análisis input-output), la teoría de Luhmann va a guardar silencio sobre este punto y no lo resolverá más que apelando a la posibilidad de conocimiento técnico que al interior de cada sistema soluciona la problemática presentada (de un modo misterioso para la teoría).

Asimismo, existe un tercer punto en el que la teoría de sistemas se diferencia de otras formas de funcionalismo o estructuralismo. Tomando por ejemplo otra vez el caso de la teoría de sistemas complejos, se sostiene que los sistemas

277 lbid., p. 19.

278 Herget, James E., Contemporary German Legal Philosophy, Philadelphia: University of Pennsylvania Press, 1996, p. 81. 
sociales, a diferencia de los biológicos, poseen propiedades emergentes que solo aparecen a nivel agregativo. Sin embargo este tipo de enfoque es propio del investigador al que le interesan los componentes de cada sistema funcional, cuestión que no es de relevancia desde la teoría de Luhmann. En esta lo que interesa son las comunicaciones que se dan al interior del sistema, y que permiten una diferenciación entre sistema y entorno con la que la supervivencia en el tiempo del sistema se asegura. Como apunta Herget:

“(...) This distinction between system and environment is an important basic concept in Luhmann's thinking. Let us now turn to the idea of social systems. How are they constituted? In most classical sociological theory the basic element of society was the individual human being, or the Weberian 'human action', or the Habermasian variant, 'communicative action'. Using one of these ideas as basic, sociologists could describe patterns of behavior, social structures, and relationships of various kinds. Luhmann rejects these traditional concepts. For him the basic component of a social system is 'communication'. Social systems process communications through their operations and structures" 279 .

Es decir que para los sistemas sociales, los individuos no son componentes sino entorno, lo que importa es la información y comunicación que fluye como conocimiento que permite la supervivencia en el tiempo del subsistema que estemos estudiando (esto es, del sistema en sí mismo). Por momentos este análisis pareciera llevar a la conclusión de que las diferencias con la propuesta de Habermas están en la base, por lo que un debate sobre otros aspectos no es siquiera necesario.

Sin embargo sí existe una forma en la que las demandas de cambio y reajuste se incorporan al sistema y es a través de la apertura cognitiva de los mis$\operatorname{mos}^{280}$. Aquí Luhmann propone que a pesar de que los sistemas sociales son

279 lbid.

280 Sobre la apertura cognitiva de los sistemas luhmannianos se han desarrollado interesantes líneas de investigación. Por ejemplo, Fernandes Campilongo en Brasil ubica al derecho como susbsistema social, y a partir de esa cartografía establece una relación entre el derecho y los movimientos sociales. Comienza para ello señalando que la tecnificación de nuestras sociedades ha llevado a un progresivo proceso de especialización funcional que no es más que la reducción de la complejidad del mundo a partir de dos variables opuestas o códigos binarios que le son relevantes a un sistema en concreto. Es decir, de la complejidad y caoticidad del mundo real, cada subsistema toma solo un elemento relevante, y a partir del mismo realiza una especialización funcional que reduce la enorme complejidad del input. El resultado es que el derecho como 
autopoiéticos en la medida de que a través de ellos circula la información y comunicación que permite su continuidad en el tiempo, los mismos necesitan adecuarse a las circunstancias mediante una apertura cognitiva. Entonces son sistemas operativamente cerrados pero cognitivamente abiertos ${ }^{281}$.

Finalmente, para Luhmann definir remite a diferenciar. Se genera un nuevo subsistema respecto del cual podemos decir que tiene categorías propias, cuando este encuentra que debido a la creciente complejización de un campo tradicional, se hace necesaria la aparición de una nueva especialización funcional. Es en ese momento en el que nace la disciplina y no antes, y es en ese momento también en el que nace la definición de dicha nueva disciplina. En clave especulativa podríamos plantear que Luhmman propone, mediante la relación de identidad entre definición y diferenciación, una solución para la colonización de la razón instrumental producida como un largo proceso luego de la caída del feudalismo. En este, un gran sistema social basado en un código binario identificable con la moral (pero también con elementos políticos) habría colapsado dejando lugar a una nueva especialización funcional basada en conocimientos técnicos. En algún momento de la historia la razón comunicativa perdió su lugar porque la complejidad del mundo la desterró como forma privilegiada de racionalidad.

susbistema se basa en el código binario legal/no legal (o en algunos otros trabajos "jurígeno/ no jurígeno"). Es decir que el derecho como subsistema habría desarrollado una compleja y sofisticada técnica hermenéutica para trabajar la materia prima de su oposición binaria, las leyes. La interpretación en el derecho, la subsunción y las jerarquías normativas toman su lugar aquí como sofisticaciones y refinamientos al interior del subsistema jurídico. Evidentemente este sistema no tiene una relación siempre pacífica con otros subsistemas como el de la política, la moral o la economía, pero es precisamente en esa tensión entre subsistemas que el derecho es capaz de incorporar elementos del entorno (recordemos que es cognitivamente abierto) para reajustar su funcionamiento. Este último punto tiene que ver con la función de los movimientos sociales como un subsistema funcional mucho más desorganizado que los de las ciencias sociales funcionalmente muy delimitadas. Las ciencias sociales tienden a establecer grandes objetivos, o buscan grandes metarelatos. Campilongo señala que el derecho busca la justicia así como la economía la eficiencia. Ahora bien, estos grandes metarelatos tienen un límite en su funcionamiento práctico. Es decir, existe una divergencia entre lo que el sistema ofrece y lo que logra. Este lugar de divergencia es asumido por los movimientos sociales como vehículos de reajuste del sistema. Si el derecho en la práctica está demasiado alejado del ideal regulativo de justicia es porque hay algo en su especialidad funcional que no da los resultados prometidos. El conflicto que ello genera se puede instrumentalizar a través de los movimientos sociales (cf. Fernandes Campilongo, Celso, Protestas sociales dentro del derecho, Lima: Pontificia Universidad Católica del Perú, Ediciones del Hipocampo, 2012).

28I Luhmann, Niklas, "Operational closure and structural coupling”, pp. 14I9-I44I. 
Este acto de destierro de la razón dialógica tuvo que ver con la misma autopoiésis del sistema social de esa época. Sin adaptación el sistema mismo colapsa y se hace anacrónico respecto de tiempos de creciente agilidad en las transacciones de mercado. En el derecho, ello tuvo un correlato en la diferenciación tajante entre derecho y moral que a inicios del siglo XX propuso Hans Kelsen ${ }^{282}$, para quien la validez del derecho corría por cuerdas totalmente separadas a las del subsistema de la moralidad. Mientras que en uno el código binario era el de validez/invalidez, en el caso de la moral era el de lo bueno/malo.

\section{\$ 2. ¿De qué forma la teoría de sistemas de Luhmann puede ser calificada como ideológica?}

Luego de la caracterización de la teoría de sistemas tal como la concibe Luhmann realizada en la sección anterior, en esta pasaremos a analizar tres puntos en los cuales Habermas podría considerar como ideológica a la teoría de sistemas $^{283}$ : la acusación de hiperracionalismo, que tiene que ver con un debate epistemológico sobre la posibilidad de conocimiento en las ciencias sociales; la acusación de fragmentación de la conciencia mediante la especialización funcional de la teoría de sistemas, y que es el campo que más propiamente tiene que ver con la concepción habermasiana de la ideología; y, finalmente, la aparición de un conservadurismo positivista en Luhmann, el mismo que se asocia a la extrapolación de categorías de las ciencias naturales para evitar el uso de las categorías de la sociología tradicional. Esta tercera crítica es muy importante porque en ella quedará claro que Habermas no puede acusar a Luhmann de hacer un uso extensivo de la categoría de la razón instrumental pues para este, el centro de la atención no está dirigido a los individuos, su conciencia o su racionalidad (así esta sea de tipo medio-fin) sino en las comunicaciones e informaciones que circulan en el sistema. Es aquí donde confrontaremos a la especialización funcional como la contracara de la razón instrumental-cognitiva, en contra de la racionalidad dialógica y moral-práctica.

282 Cf. Kelsen, Hans, Teoría pura del derecho: introducción a los problemas de la ciencia jurídica, Madrid: Trotta, 2011.

283 Ello sigue en alguna medida el estudio de Luis Armando González sobre la disputa Habermas-Luhmann (cf. González, Luis, Teoría critica versus teoría de sistemas: La confrontación Habermas-Luhmann, disponible en: http://www.uca.edu.sv/revistarealidad/archivo/4e2d8da2d 86eateoriacritica.pdf). 
Entonces, respecto al primer punto de crítica, retomar la influencia del debate Adorno-Popper en la obra de Habermas resulta de utilidad. El hiperracionalismo aparece en el momento en el que el científico social acusa cientificismo en su labor y considera que es posible la extrapolación del método inductivo a las ciencias sociales, y, más aun, a aquellos espacios en los que es necesario que opere una deliberación regida normativamente por una situación ideal del habla ${ }^{284}$.

Si bien la explicación de la teoría de los sistemas parece dar cuenta plausible de los procesos de tecnificación del mundo, su puro diagnóstico carece de elementos críticos suficientes como para cuestionar una forma parcial de racionalidad. Luhmann entonces, sin decirlo expresamente, está suponiendo que la progresiva complejización del mundo es un proceso sin marcha atrás en la medida que la racionalización del mundo solo es posible respecto de un modelo instrumental-cognitivo. De esta forma le serían aplicables todas las críticas que Habermas dirigió contra Weber y la sociología clásica de la acción racional con arreglo a fines. La miopía hiperracionalista, finalmente, tiene que ver con la incapacidad de Luhmann para notar que en paralelo al proceso de especialización funcional del mundo se desenvuelve un proceso de colonización del mundo de la vida pues su especialidad funcional no queda clara (a grandes rasgos, el papel limitado de la política en el modelo de Luhmann se explica por estas mismas razones ${ }^{285}$ ).

En segundo lugar, con Habermas podemos sostener que la especialización funcional en cada vez más subsistemas genera la fragmentación de la conciencia y de esta manera nos encontramos frente a la forma prototípica de la ideología que Habermas identificó en Conocimiento e interés. La fragmentación parte del tratamiento aparentemente científico que fetichiza los resultados obtenidos, haciéndolos inmunes a la crítica. Para graficar esta crítica de Habermas podemos

284 Rodríguez lo grafica en estos términos: "Así lo comprendió Habermas, quien (...) siempre concibe la tarea de una teoría crítica de la sociedad como proceso entre aquello susceptible de regirse por las reglas de la autorreflexividad (lo intersubjetivo o plenamente comunicativo) y aquello otro regido sin paliativos por reglas técnicas (el mundo de los sistemas)" (Rodríguez, José Enrique, "La crítica de la modernidad”, en: Giner, Salvador (coord.), Teoría sociológica moderna, 2da. edición, Barcelona: Ariel, 20II, p. 657).

285 Cf. Luhmann, Niklas, "Politicians, Honesty and the Higher Amorality of Politics", disponible en: http://tcs.sagepub.com 
recordar los debates públicos sobre reformas de sistemas prestacionales por parte de los Estados de bienestar modernos. Uno de los bandos de respuestas a las demandas de ampliación prestacional en salud o educación es que dichos reclamos no se basan en criterios técnicos sostenibles. El resultado natural de esta reducción argumental es el acto de desechar las demandas debido a su imprecisión técnica. Solo mediante una recuperación o repolitización de los procesos de toma de decisiones que involucran a toda la sociedad se logra que se levanten las restricciones impuestas a la racionalidad comunicativa y la censura al discurso no técnico desaparezca. Aquí podemos volver sobre una de las aplicaciones psicoanalíticas que Ricoeur creía posible extrapolar a la teoría crítica: de lo que se trata no es de mejorar la comunicación, pues el problema de la lucha de clases en clave deliberativa tiene que ver con una colonización que está en la base del diagnóstico de la época. Si el problema es el de la racionalización, entonces los paliativos no surten efectos. En clave contextual, se podrán incorporar nuevas partes en uno u otro debate público concreto, ello en atención a presiones populares, pero la tendencia a largo plazo consistente en la tecnificación de las decisiones no va a desaparecer, $y$, más aun, es probable que siguiendo el modelo adaptativo de Luhmann, opere mediante mecanismos cada vez más refinados.

El remedio entonces, y entrando al plano de la tercera crítica que Habermas podría dirigir contra Luhmann, más que consistir en el camino de la especialización funcional, consiste en el camino inverso, en la complejización de las variables mediante un debate público abierto en el que se busque la participación basada en un modelo ideal del habla. Evidentemente este modelo tiene sus restricciones pues, en primera instancia, parece que no siempre las decisiones sometidas a deliberación son las que mejor se toman. Aun así, dos precisiones se pueden hacer al respecto: la primera es que ni Habermas consiente en que una situación ideal del habla consista en un modelo democrático meramente agregativo (ello queda claro a partir de su visión de derechos individuales presentada en Facticidad y validez), y, en segundo lugar, Luhmann mismo reconoce que el campo de interacción entre diversos subsistemas y el sistema global de la sociedad es complejo, usualmente conflictivo y en el que suelen producirse grandes pérdidas de eficiencia ${ }^{286}$, por lo que la democracia abierta 
podría ser una opción igual de costosa que la mayor especialización funcional. Así, a pesar de la renuncia al uso de categorías de análisis relacionadas a los sujetos en la obra de Luhmann, pareciera que al fondo subyace un trabajo de racionalidad instrumental-cognitiva. Para percibir ello debemos dejar de lado que la teoría de Luhmann no se refiere a los individuos sino a los sistemas para solo percibir el aspecto formal del medio-fin. Así, al igual que la sociología tradicional estudia los medios por los cuales los individuos consiguen cosas o alcanzan objetivos, la teoría de sistemas estudia las formas por las cuales los sistemas consiguen sobrevivir en el tiempo. La supervivencia tiene que ver con sofisticados mecanismos adaptativos a los que ya antes hemos hecho referencia como la apertura cognitiva, la autopoiésis o el acoplamiento del entorno, pero en cualquier caso se trata de la misma racionalidad instrumental que ha sido encubierta mediante un objeto de estudio distinto: la sociedad. El conservadurismo ahora aparece más claro: Luhmann es conservador porque considera que los métodos de estudio de las ciencias naturales son extrapolables por reificación a los procesos de los sistemas sociales. Logramos aplicar la biología a los sistemas sociales solo cuando vemos en ellos elementos de vida que no pueden depender solamente de la información que circula a su interior. En ese sentido, desconocer que quienes conforman dichos sistemas son individuos de carne y hueso encubre el hecho de que la asociación en sociedades tuvo en sus orígenes una finalidad que no estaba asociada a la autonomía misma del sistema, sino con consensos logrados dialógicamente ${ }^{287}$. Ese espacio, sin embargo, se ha comenzado a perder a causa de la colonización de la razón instrumental.

En este aspecto hay una similitud con la crítica que Habermas formuló contra el marxismo:

"En este libro [Conocimiento e interés] lo más interesante es la manera en que el autor observa en el marxismo clásico un sesgo tecnicista. Consiste este sesgo en concebir el conflicto básico de la sociedad como un conflicto que tiene lugar exclusivamente entre estructuras $y$ sujetos de un único plano de la actividad social -el de la producción en sentido estrecho o meramente material-, olvidando que el conflicto permanente entre estructuras y sujetos tiene lugar también en el seno de la producción simbólica (o aspecto intersubjetivo y comunicativo

287 De hecho, la tradición contractualista en filosofía política se basa en este presupuesto. 
de la sociedad). La dialéctica de Marx, en una palabra, describirá muy bien los conflictos del homo faber pero no los del homo loquens" ${ }^{288}$.

Por lo tanto, es necesario recuperar el espacio de la interacción simbólica entre individuos pues por virtud de las categorías analíticas del marxismo, o también de la teoría de sistemas, se ha perdido de vista a los conformantes de las estructuras que se analizan. Era por ello por lo que Habermas, por ejemplo en su Teoría de la acción comunicativa primero se acerca y luego renuncia a los modelos de Dilthey y Peirce por considerarlos culturalistas, y adopta un punto medio que encuentra en Freud y consistente en trasladar categorías psicoanalíticas al ámbito social.

\section{§ 3. Repolitizando el debate público: algunas conclusiones}

El hilo conductor de esta investigación ha sido el responder a la pregunta de en qué sentido Jürgen Habermas retoma la tradición de crítica a formas ideológicas de pensamiento - de génesis marxista- en su debate con la teoría de sistemas de Niklas Luhmann. Para responder a esta interrogante recorrimos la evolución teórica que va desde la formulación de los elementos de la crítica a la ideología en el propio Marx, hasta la reinterpretación que hace Habermas de esta crítica incorporando elementos de la teoría crítica y del psicoanálisis.

Finalmente, podemos responder a la pregunta planteada inicialmente señalando que Habermas retoma la crítica a la ideología marxista caracterizando al pensamiento de Luhmann como una forma de positivismo ideológico. Para el logro de este objetivo, Habermas coloca en el centro de la atención de una gran teoría social a los individuos -mediante su teoría de la acción comunicativapues las comunicaciones al interior de los sistemas son inviables sin individuos que, a su vez, se relacionen con dichos sistemas.

Así, como hemos visto, mediante el mantenimiento de la categoría de individuos que actúan en sociedad, Habermas se premune del cientificismo que apareció como uno de los puntos más importantes del debate Popper-Adorno

288 Rodríguez, José Enrique, “La crítica de la modernidad”, en: Giner, Salvador (coord.), op.cit., p. 667. 
durante el siglo pasado. Solo en ese sentido, además, se pudo percibir que la renuncia a los individuos implica una aparición, pero ahora por la trastienda, de la racionalidad instrumental en Luhmann, quien en principio niega y desprecia dichas categorías analíticas. 